\title{
Genome-Wide Fetal Aneuploidy Detection by Maternal Plasma DNA Sequencing
}

\author{
Diana W. Bianchi, MD, Lawrence D. Platt, MD, James D. Goldberg, MD, Alfred Z. Abuhamad, MD, \\ Amy J. Sehnert, MD, and Richard P. Rava, PhD, on behalf of the MatErnal BLood IS Source to \\ Accurately diagnose fetal aneuploidy (MELISSA) Study Group*
}

OBJECTIVE: To prospectively determine the diagnostic accuracy of massively parallel sequencing to detect whole chromosome fetal aneuploidy from maternal plasma.

METHODS: Blood samples were collected in a prospective, blinded study from 2,882 women undergoing prenatal diagnostic procedures at 60 U.S. sites. An independent biostatistician selected all singleton pregnancies with any abnormal karyotype and a balanced number of randomly selected pregnancies with euploid karyotypes. Chromosome classifications were made for each sample

"For a list of study sites that participated in the MELISSA study, see the
Appendix online at http://links.lwer.com/AOG/A290.

From the Mother Infant Research Institute, Tufts Medical Center and Tufts University School of Medicine, Boston Massachusetts; the Center for Fetal Medicine and Women's Ultrasound and the David Geffen School of Medicine, the University of California, Los Angeles, Los Angeles, California; the Prenatal Diagnosis Center, San Francisco Perinatal Associates, San Francisco, California; the Eastern Virginia Medical School, Norfolk, Virginia; and Verinata Health, Inc., Redwood City, California.

Funded by Verinata Health, Inc., Redwood City, California.

The authors thank the pregnant women who enrolled in this study and the center staff at the participating sites, without whom this research could not be conducted; the expertise and contributions of Dr. Philip Cotter for performing karyotype classifications; Andrea Rowe, Senior Biostatistician at Quintiles; April Ruby and Kelly Oliver for their lead roles in clinical operations and overseeing site and data management; all members of the Verinata Health laboratory staff for processing, sequencing, and data processing of the samples; and the clinical research and clinical research organization teams for site recruitment, coordination, and monitoring.

Presented at the Society for Maternal-Fetal Medicine $32^{\text {nd }}$ annual meeting, February 6-11, 2012, Dallas, Texas.

Corresponding author: Richard P. Rava, 800 Saginaw Drive, Redwood City, CA 94063; e-mail: rrava@verinata.com.

\footnotetext{
Financial Disclosure

Drs. Bianchi, Platt, and Abuhamad received honoraria for their roles on the Verinata Health, Inc. Clinical Advisory Board and hold equity in the company. Dr. Goldberg received honoraria for his role on the Verinata Health Clinical Advisory Board. Dr. Sehnert is an employee of Verinata Health, Inc. Dr. Rava is the President and Co-Scientific Founder of Verinata Health.

(C) 2012 by The American College of Obstetricians and Gynecologists. Published by Lippincott Williams \& Wilkins.

ISSN: 0029-7844/12
}

by massively parallel sequencing and compared with fetal karyotype.

RESULTS: Within an analysis cohort of 532 samples, the following were classified correctly: 89 of 89 trisomy 21 cases (sensitivity 100\%, 95\% [confidence interval] $\mathrm{Cl}$ 95.9-100), 35 of 36 trisomy 18 cases (sensitivity $97.2 \%$, 95\% Cl 85.5-99.9), 11 of 14 trisomy 13 cases (sensitivity 78.6\%, 95\% Cl 49.2-99.9), 232 of 233 females (sensitivity $99.6 \%, 95 \% \mathrm{Cl} 97.6$ to more than 99.9 ), 184 of 184 males (sensitivity $100 \%, 95 \% \mathrm{Cl} 98.0-100$ ), and 15 of 16 monosomy $X$ cases (sensitivity $93.8 \%, 95 \% \mathrm{Cl} 69.8-99.8$ ). There were no false-positive results for autosomal aneuploidies $(100 \%$ specificity, $95 \% \mathrm{Cl}$ more than 98.5 to 100$)$. In addition, fetuses with mosaicism for trisomy $21(3 / 3)$, trisomy $18(1 / 1)$, and monosomy $X(2 / 7)$, three cases of translocation trisomy, two cases of other autosomal trisomies (20 and 16), and other sex chromosome aneuploidies (XXX, XXY, and XYY) were classified correctly.

CONCLUSION: This prospective study demonstrates the efficacy of massively parallel sequencing of maternal plasma DNA to detect fetal aneuploidy for multiple chromosomes across the genome. The high sensitivity and specificity for the detection of trisomies 21, 18, 13, and monosomy $X$ suggest that massively parallel sequencing can be incorporated into existing aneuploidy screening algorithms to reduce unnecessary invasive procedures.

CLINICAL TRIAL REGISTRATION: ClinicalTrials.gov, www.clinicaltrials.gov, NCT01122524.

(Obstet Gynecol 2012;119:00-00)

DOI: 10.1097/AOG.0b013e31824fb482

LEVEL OF EVIDENCE: II

$\mathrm{n}$ most developed countries, prenatal screening algorithms for aneuploidy involve measurement of multiple analytes in maternal serum combined with ultrasonographic measurement of the fetal nuchal translucency. Initially developed to detect neural tube defects, these serum screening protocols have been expanded to detect trisomy 21 (T21, Down syn- 
drome) and trisomy 18 (T18, Edwards syndrome). ${ }^{1,2}$ Although these algorithms have been validated extensively and are accepted widely, they are not used universally to detect trisomy 13 (T13, Patau syndrome) and they do not detect monosomy X (45, X, Turner syndrome). Definitive diagnosis of fetal chromosomal aneuploidies still requires the performance of invasive procedures such as chorionic villus sampling (CVS) or amniocentesis, which are associated with a risk of miscarriage.

Advances in the technology for sequencing cell free DNA in maternal plasma have enabled precise molecular counting of chromosome copy numbers. ${ }^{3-15}$ The majority of research in this area has been conducted using retrospectively collected sample biobanks. ${ }^{10,12,14}$ To date, the only published prospective clinical study focused exclusively on classification of trisomy 21 compared with samples with normal karyotypes..$^{15}$ In the current study, we report on a prospective, blinded study that more closely emulates an actual population of pregnant women in whom the fetal karyotype is unknown, and all samples with any abnormal karyotypes were selected for sequencing. The sequencing classifications were compared with fetal karyotypes from invasive procedures to determine the diagnostic performance of massively parallel sequencing for multiple chromosomal aneuploidies.

\section{MATERIALS AND METHODS}

The MELISSA (MatErnal BLood IS Source to Accurately diagnose fetal aneuploidy) study was conducted as a prospective, multicenter observational study with blinded nested case-control analyses. Pregnant women 18 years and older undergoing an invasive prenatal procedure to determine fetal karyotype were recruited to participate (Clinicaltrials.gov NCT01122524). Eligibility criteria included pregnant women between 8 0/7 and 22 weeks $0 / 7$ weeks of gestation who met at least one of the following additional criteria: age 38 years or older, positive screening test result for fetal aneuploidy (by serum analytes, nuchal translucency measurement, or both), presence of ultrasound markers associated with an increased risk for fetal aneuploidy, or a prior aneuploid fetus. Written informed consent was obtained from all women who agreed to participate.

Enrollment occurred at 60 geographically dispersed medical centers in 25 states per protocol approved by institutional review boards at each institution. Two clinical research organizations (Quintiles, Durham, NC, and Emphusion, San Francisco, CA) were retained to maintain study blinding and provide clinical data management, data monitoring, biostatistics, and data analysis services.
Before any invasive procedure was performed, a peripheral venous blood specimen $(17 \mathrm{~mL})$ was collected in two acid citrate dextrose tubes that were de-identified and labeled with a unique study number. Site research personnel entered study number, date, and time of blood draw into a secure electronic case report form. Whole blood samples were shipped overnight in temperature-controlled containers from sites to the Verinata Health laboratory (then in San Carlos, CA) (Fig. 1). On receipt and sample inspection, cell-free plasma was prepared per previously described methods ${ }^{11}$ and stored frozen at $-80^{\circ} \mathrm{C}$ in 2 to 4 aliquots until time of sequencing. Date and time of sample receipt at Verinata Health were recorded. A sample was determined to be eligible for analysis if it was received overnight, was cool to touch, and contained at least $7 \mathrm{~mL}$. Samples that were eligible at receipt were reported to the clinical research organization weekly and used for selection on a random sampling list (see below and Fig. 2). Clinical data from the woman's current pregnancy and fetal karyotype were entered into the electronic case report form by site research personnel and verified by clinical research organization monitors through source document review.

Sample size determination was based on the precision of the estimates for a targeted range of performance characteristics (sensitivity and specificity) for the index test. Specifically, the number of affected (T21, T18, T13, male, female, or monosomy $\mathrm{X}$ ) cases and unaffected (non-T21, non-T18, non-T13, not male, not female, or not monosomy $\mathrm{X}$ ) controls were determined to estimate the sensitivity and specificity, respectively, to within a prespecified margin of error based on the normal approximation $(\mathrm{n}=(1 \cdot 96$ $\checkmark P(1-P) /$ margin of error $)^{2}$, where $P=$ the estimate of the sensitivity or specificity). Assuming a true sensitivity of $95 \%$ or greater, a sample size between 73 to 114 cases ensured that the precision of the estimate of sensitivity would be such that the lower bound of the $95 \%$ confidence interval (CI) would be $90 \%$ or greater (margin of error $5 \%$ or less). For smaller sample sizes, a larger estimated margin of error of the $95 \%$ CI for sensitivity was projected (from $6 \%$ to $13.5 \%$ ). To estimate the specificity with greater precision a larger number of unaffected controls ( $\sim 4: 1$ ratio to cases) was planned at the sampling stage. This ensured the precision of the estimate of specificity to at least $3 \%$. Using this method, as the sensitivity, specificity, or both increased, the precision of the CI also would increase.

Based on sample size determination, a random sampling plan was devised for the clinical research 


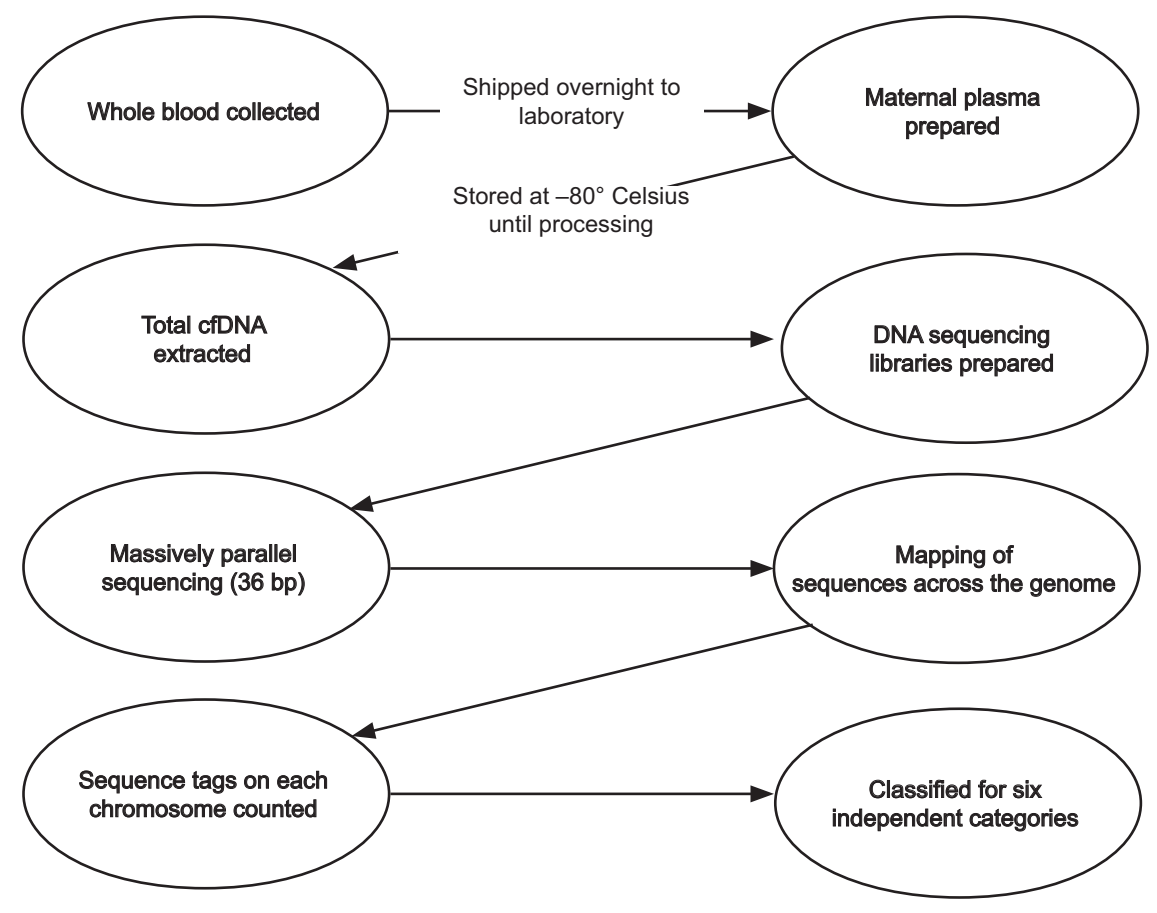

Fig. 1. Laboratory process flow for classifying samples using massively parallel sequencing.

Bianchi. Genome-Wide Fetal Aneuploidy Detection. Obstet Gynecol 2012. organization to generate lists of selected samples to sequence (minimum of 110 total samples affected by T21, T18, and T13, and 400 nonaffected samplesallowing up to half of these to have karyotypes other than $46, \mathrm{XX}$ or $46, \mathrm{XY}$ ). Women with a singleton pregnancy and a qualified blood sample were eligible for selection. Those with ineligible samples, no karyotype recorded, or a multiple gestation were excluded (Fig. 2). Lists were generated on a regular basis throughout the study and sent to the Verinata Health laboratory.

Each eligible blood sample was analyzed for six independent categories. The categories were aneuploidy status for chromosomes 21, 18, and 13 and sex status for male, female, and monosomy $\mathrm{X}$. While still blinded, the massively parallel sequencing laboratory prospectively generated one of three classifications (affected, unaffected, or unclassified) for each of the six independent categories for each plasma DNA sample. Using this scenario, the same sample could be classified as affected in one analysis (eg, aneuploidy for chromosome 21) and unaffected for another analysis (eg, euploid for chromosome 18).

Conventional metaphase cytogenetic analysis of cells obtained by CVS or amniocentesis was used as the reference standard in this study. Fetal karyotyping was performed in diagnostic laboratories routinely used by the participating sites. If after enrollment a patient underwent both CVS and amniocentesis, karyotype results from amniocentesis were used for study analysis. Fluorescence in situ hybridization results for chromosomes 21, 18, 13, $\mathrm{X}$, and $\mathrm{Y}$ were allowed if a metaphase karyotype was not available (Table 1). All abnormal karyotype reports (ie, other than $46, \mathrm{XX}$ and $46, \mathrm{XY}$ ) were reviewed by a boardcertified cytogeneticist and classified as affected or unaffected with respect to chromosomes 21,18 , and 13 and sex status for XX, XY, and monosomy X.

Prespecified protocol conventions defined the following complex abnormal karyotypes to be assigned a status of "censored" for karyotype by the cytogeneticist: triploidy, tetraploidy, complex karyotypes other than trisomy (eg, mosaicism) that involved chromosomes 21,18 , or 13 , mosaics with mixed sex chromosomes, sex chromosome aneuploidy or karyotypes that could not be fully interpreted by the source document (eg, marker chromosomes of unknown origin). Because the cytogenetic diagnosis was not known to the sequencing laboratory, all cytogenetically censored samples were analyzed independently and assigned a massively parallel sequencing classification but not included in the statistical analysis. Censored status pertained only to the relevant one or more of the six analyses (eg, a mosaic T18 would be censored from chromosome 18 analysis, but considered "unaffected" for other analyses, such as chromosomes 21, 13, X, and Y) (Table 2). Other abnormal and rare complex karyotypes, which could not be fully anticipated at the time of protocol design, were not censored from analysis (Table 3). 


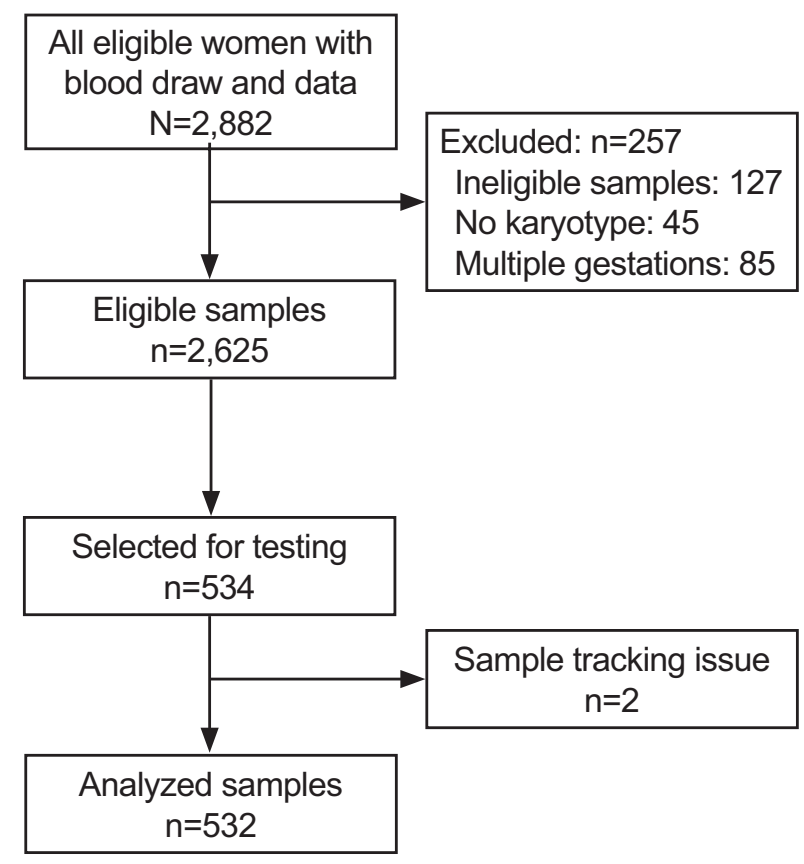

A

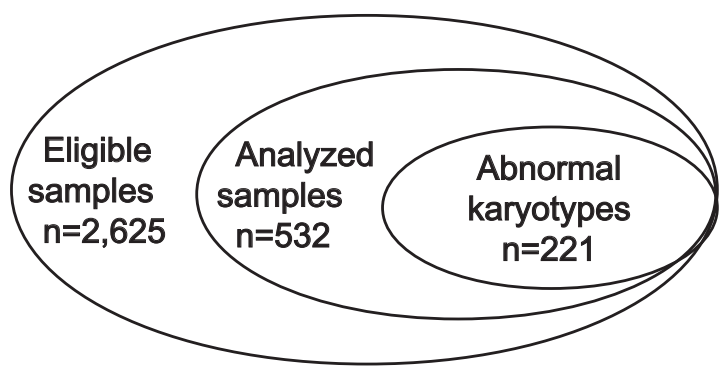

B

Fig. 2. Study design (A) and random sampling plan (B). Bianchi. Genome-Wide Fetal Aneuploidy Detection. Obstet Gynecol 2012.

The data contained in the electronic case report form and clinical database were restricted to authorized users only (at the study sites, clinical research organizations, and contract clinical personnel). It was not accessible to any employees at Verinata Health until the time of unblinding.

After receiving random sample lists from the clinical research organization, total cell-free DNA (a mixture of maternal and fetal) was extracted from thawed selected plasma samples using a QIAamp DNA Blood Mini Kit according to the manufacturer's instructions as described previously ${ }^{11}$ (Fig. 1). In this study, sequencing libraries were prepared using the
Illumina TruSeq kit v2.5 and sequencing (6-plex, ie, 6 samples/lane) was performed on an Illumina HiSeq 2000 instrument in the Verinata Health laboratory. Single-end reads of 36 base pairs were obtained.

The clinical protocol required evidence of fetal DNA presence to report a classification result. A massively parallel sequencing classification of male or aneuploid was considered sufficient evidence of fetal DNA. In addition, each sample was also tested for the presence of fetal DNA sequentially using two allele specific methods. In the first method, the AmpflSTR Minifiler kit was used to interrogate the presence of a fetal component in the cell free DNA. Electrophoresis of short tandem repeat amplicons was carried out on the ABI 3130 Genetic Analyzer following manufacturer's protocols. All nine short tandem repeat loci in this kit were analyzed by comparing the intensity of each peak reported as a percentage of the sum of the intensities of all peaks, and the presence of minor peaks was used to provide evidence of fetal DNA. In cases in which no minor short tandem repeat could be identified, an aliquot of the sample was examined with a single nucleotide polymorphism panel of 15 single nucleotide polymorphisms with average heterozygosity of 0.4 or more selected from the Kidd et al panel. ${ }^{16}$

The sequence mapping process was identical to our previous work. ${ }^{11}$ Only reads that unambiguously mapped to a single genomic location were included. Genomic sites where reads mapped were counted and included in the calculation of normalized chromosome values. The classification algorithm in the current study was also identical to that previously reported $^{11}$; however, because the sequencing in this study was carried out on a different instrument than our previous work with multiple samples per lane, new normalizing chromosome denominators had to be determined. The normalizing chromosome denominators in the current study were determined based on a training set of 110 independent unaffected samples (ie, not from MELISSA-eligible samples) sequenced before analysis of the study samples. The new normalizing chromosomes denominators were determined by calculating all possible permutations of denominators for all autosomes and sex chromosomes that minimized the variation for the unaffected training set for all chromosomes across the genome. In the current study, the new normalized chromosome values had a higher precision than in our previous work for unaffected samples. For classification of the autosomal aneuploidy, we required a normalized chromosome value more than 4.0 to classify the chromosome as affected (ie, aneuploid for that chromosome) and a normalized chromosome 


\begin{tabular}{|c|c|c|c|}
\hline & $\begin{array}{l}\text { Eligible Patients } \\
\qquad(\mathrm{N}=2,882)\end{array}$ & $\begin{array}{l}\text { Analyzed Patients } \\
\qquad(\mathrm{n}=534)\end{array}$ & $\begin{array}{l}\text { Affected Patients } \\
\qquad(n=221)\end{array}$ \\
\hline \multicolumn{4}{|l|}{ Maternal age (y) } \\
\hline Mean \pm SD & $35.8 \pm 5.93$ & $35.2 \pm 6.40$ & $34.4 \pm 6.73$ \\
\hline Min-max & $18-49$ & $18-46$ & $18-46$ \\
\hline Multiparous & $2,348(81.5)$ & $425(79.5)$ & $176(79.6)$ \\
\hline Pregnancy by assisted reproductive techniques & $247(8.6)$ & $38(7.1)$ & $17(7.7)$ \\
\hline \multicolumn{4}{|l|}{ Race } \\
\hline White & $2,078(72.1)$ & $388(72.7)$ & $161(72.9)$ \\
\hline African American & $338(11.7)$ & $58(10.9)$ & $28(12.7)$ \\
\hline Asian & $271(9.4)$ & $53(9.9)$ & $18(8.1)$ \\
\hline Native American or Alaska Native & $22(0.8)$ & $5(0.9)$ & $2(0.9)$ \\
\hline Multiracial & $173(6.0)$ & $30(5.6)$ & $12(5.4)$ \\
\hline \multicolumn{4}{|l|}{$\mathrm{BMI}\left(\mathrm{kg} / \mathrm{m}^{2}\right)$} \\
\hline Mean \pm SD & $26.6 \pm 5.89$ & $26.2 \pm 5.73$ & $26.2 \pm 5.64$ \\
\hline Min-max & $15-76$ & $17-59$ & $18-56$ \\
\hline Current smoker & $165(5.7)$ & $29(5.4)$ & $6(2.7)$ \\
\hline Maternal diabetes mellitus & $61(2.1)$ & $11(2.1)$ & $6(2.7)$ \\
\hline \multicolumn{4}{|l|}{ Trimester } \\
\hline First & 832 (28.9) & $165(30.9)$ & $126(57.0)$ \\
\hline Second & $2,050(71.1)$ & $369(69.1)$ & $95(43.0)$ \\
\hline \multicolumn{4}{|l|}{ Gestational age* (wk) } \\
\hline Mean $\pm S D$ & $15.5 \pm 3.27$ & $15.1 \pm 3.16$ & $14.8 \pm 3.18$ \\
\hline Min-max & $8-31$ & $10-23$ & $10-23$ \\
\hline \multicolumn{4}{|l|}{ Karyotype source } \\
\hline CVS & $1,044(36.8)$ & $228(42.7)$ & $121(54.8)$ \\
\hline Amniocentesis & $1,783(62.8)$ & $301(56.4)$ & $95(43.0)$ \\
\hline Products of conception & $10(0.4)$ & $5(0.9)$ & $5(2.2)$ \\
\hline Amniocentesis after CVS & $7(0.2)$ & $1(0.2)$ & $0(0.0)$ \\
\hline Karyotype by FISH-only & $105(3.6)$ & $18(3.4)$ & $13(5.9)$ \\
\hline \multicolumn{4}{|l|}{ No. of fetuses } \\
\hline 1 & $2,797(97.1)$ & $534(100)$ & $221(100)$ \\
\hline 2 & $76(2.6)$ & $0(0.0)$ & $0(0.0)$ \\
\hline 3 & $7(0.2)$ & $0(0.0)$ & $0(0.0)$ \\
\hline 4 & $2(0.2)$ & $0(0.0)$ & $0(0.0)$ \\
\hline \multicolumn{4}{|l|}{ Prenatal risk } \\
\hline Advanced maternal age only (age 38 y or older) & $1,061(36.8)$ & $152(28.5)$ & $21(9.5)$ \\
\hline Positive screen risk & $622(21.6)$ & $91(17.0)$ & $14(6.3)$ \\
\hline Ultrasound abnormality & $477(6.6)$ & $122(22.8)$ & $81(36.7)^{+}$ \\
\hline Prior aneuploid pregnancy & $82(2.8)$ & $15(2.8)$ & $4(1.8)$ \\
\hline More than 1 risk & $640(22.2)$ & $154(28.9)$ & $101(45.7)^{\dagger}$ \\
\hline Screening risk estimated by & 1,749 & 310 & 125 \\
\hline Nuchal translucency measure alone & $179(10.2)$ & $53(17.1)$ & $36(28.8)$ \\
\hline First-trimester combined & $677(38.7)$ & $117(37.7)$ & $47(37.6)$ \\
\hline Second-trimester triple or quadruple & $414(23.7)$ & $72(23.3)$ & $16(12.8)$ \\
\hline Fully integrated (first and second trimester) & $137(7.8)$ & $14(4.5)$ & $3(2.4)$ \\
\hline Sequential & $218(12.5)$ & $32(10.3)$ & $15(12.0)$ \\
\hline Other & $124(7.1)$ & $22(7.1)$ & $8(6.4)$ \\
\hline Abnormal fetal ultrasound scan & $837(29.0)$ & $242(45.3)$ & $166(75.1)^{+}$ \\
\hline One or more soft marker & $719(24.9)$ & $212(39.7)$ & $143(64.7)$ \\
\hline One or more major marker & $228(7.9)$ & $79(15.8)$ & $65(29.4)$ \\
\hline IUGR (less than $10^{\text {th }}$ percentile) & $26(0.9)$ & $11(2.1)$ & $11(5.0)$ \\
\hline Amniotic fluid volume abnormality & $24(0.8)$ & $7(1.3)$ & $4(1.8)$ \\
\hline
\end{tabular}

SD, standard deviation; BMI, body mass index; CVS, chorionic villus sampling; FISH, fluorescence in situ hybridization; IUGR, intrauterine growth restriction.

Data are $\mathrm{n}(\%)$ unless otherwise specified.

* Gestational age at time of invasive procedure.

${ }^{+}$Higher penetrance of ultrasound abnormalities in fetuses with abnormal karyotypes. 


\begin{tabular}{|c|c|c|c|}
\hline \multirow[b]{2}{*}{ Karyotype } & \multirow{2}{*}{$\begin{array}{l}\text { Censored } \\
\text { Category }\end{array}$} & \multicolumn{2}{|c|}{$\begin{array}{c}\text { Massively Parallel } \\
\text { Sequencing Classification }\end{array}$} \\
\hline & & Aneuploidy & Sex \\
\hline \multicolumn{4}{|l|}{ Mosaic trisomy 21 and $18(n=4)$} \\
\hline $47, \mathrm{XY},+21[5] / 46, \mathrm{XY}[12]$ & 21 & Affected (T21) & Male \\
\hline $47, X X,+21[4] / 46, X X[5]$ & 21 & Affected (T21) & Unclassified \\
\hline $47, X Y,+21[21] / 48, X Y,+21+\operatorname{mar}[4]^{*}$ & $21,18,13$, sex & Affected (T21) & Male \\
\hline $47, \mathrm{XX},+18[42] / 46, \mathrm{XX}[8]$ & 18 & Affected (T18) & Female \\
\hline \multicolumn{4}{|l|}{ Other complex mosaicism $(n=2)$} \\
\hline 45,XY,-13[5]/46,XY,r (13) (p11.1q22)[15] & 13 & Unaffected $(21,18,13)$ & Male \\
\hline $92, X X X X[20] / 46, X X[61]$ & $21,18,13$, sex & Unaffected $(21,18,13)$ & Unclassified \\
\hline \multicolumn{4}{|l|}{ Added material of uncertain origin $(n=5)$} \\
\hline $46, X X$, add $(X)(p 22.1)$ & $21,18,13$, sex & Unaffected $(21,18,13)$ & Female \\
\hline $46, X Y$, add $(10)(q 26)$ & $21,18,13$, sex & Unaffected $(21,18,13)$ & Male \\
\hline $46, X Y$, add (15) (p11.2) & $21,18,13$, sex & Unaffected $(21,18,13)$ & Male \\
\hline $47, \mathrm{XY},+\operatorname{mar} / 46, \mathrm{XY}$ & $21,18,13$, sex & Unaffected $(21,18,13)$ & Male \\
\hline $47, \mathrm{XX},+\operatorname{mar}[12] / 46, \mathrm{XX}[8]$ & $21,18,13$, sex & Unaffected $(21,18,13)$ & Female \\
\hline \multicolumn{4}{|l|}{ Triploidy $(n=10)$} \\
\hline $69, X X Y$ & $21,18,13$, sex & Unaffected $(21,18,13)$ & Unclassified sex \\
\hline \multirow[t]{2}{*}{$69, X X X(n=9)$} & $21,18,13$, sex & Unaffected $(21,18,13)(n=6)$ & Female $(n=5)$ \\
\hline & & No fetal DNA detected $(n=3)$ & $\begin{array}{l}\text { No fetal DNA detected }(n=3) \\
\text { Unclassified }(n=1)\end{array}$ \\
\hline \multicolumn{4}{|l|}{ Sex chromosome aneuploidy $(n=10)$} \\
\hline $47, X X X(n=4)$ & Sex & Unaffected $(21,18,13)(n=4)$ & $\begin{array}{l}X X X(n=3) \\
\text { Monosomy X }(n=1)\end{array}$ \\
\hline $47, X X Y(n=2)$ & Sex & Unaffected $(21,18,13)(\mathrm{n}=2)$ & $X X Y(n=2)$ \\
\hline $48, X X Y,+18$ & & $\begin{array}{l}\text { Unclassified }(18) \text { and } \\
\text { Unaffected }(21,13)(\mathrm{n}=1)\end{array}$ & Unclassified $(\mathrm{n}=1)$ \\
\hline $47, X Y Y(n=3)$ & Sex & Unaffected $(21,18,13)(n=3)$ & $X Y Y(n=3)$ \\
\hline \multicolumn{4}{|l|}{ Mosaic monosomy $X(n=7)$} \\
\hline $45, X / 46, X X(n=3)$ & Sex & Unaffected $(21,18,13)(n=3)$ & $\begin{array}{l}\text { Female }(n=2) \\
\text { Monosomy X }(n=1)\end{array}$ \\
\hline $45, X / 47, X X X(n=1)$ & Sex & Unaffected $(21,18,13)$ & Monosomy X \\
\hline $45, X / 46, X Y(n=2)$ & Sex & Unaffected $(21,18,13)(n=2)$ & Male $(n=2)$ \\
\hline $\begin{array}{l}45, X,+21, \operatorname{der}(14 ; 21)(q 10 ; q 10)[4] / \\
46, X Y,+21, \operatorname{der}(14 ; 21)(q 10 ; q 10)[17]\end{array}$ & Sex & $\begin{array}{l}\text { Affected (T21) and } \\
\text { Unaffected }(18,13)\end{array}$ & Male \\
\hline \multicolumn{4}{|l|}{ Other reasons $(n=3)$} \\
\hline Sex not disclosed in report $(n=2)$ & Sex & Unaffected $(21,18,13)$ & Female $(n=2)$ \\
\hline $46, X Y$ with maternal cell contamination $(n=1)$ & Sex & Unaffected $(21,18,13)$ & Male \\
\hline
\end{tabular}

* Patient excluded from all analysis categories owing to marker chromosome in one cell line.

value less than 2.5 to classify a chromosome as unaffected. Samples with autosomes that have a normalized chromosome value between 2.5 and 4.0 were called "unclassified."

Sex chromosome classification was performed in a more complex fashion-by sequential application of normalized chromosome values for both $\mathrm{X}$ and $\mathrm{Y}$. Specifically:

1. If normalized chromosome value $\mathrm{X}<-4.0$ and normalized chromosome value $\mathrm{Y}<2.5$, the sample was classified as monosomy $\mathrm{X}$.

2. If normalized chromosome value $\mathrm{X}>-2.5$ and normalized chromosome value $\mathrm{X}<2.5$ and normalized chromosome value $\mathrm{Y}<2.5$, the sample was classified as female (XX).
3. If normalized chromosome value $X>4.0$ and normalized chromosome value $\mathrm{Y}<2.5$, the sample was classified as XXX.

4. If normalized chromosome value $\mathrm{X}>-2.5$ and normalized chromosome value $\mathrm{X}<2.5$ and normalized chromosome value $\mathrm{Y}>33$, the sample was classified as XXY.

5. If normalized chromosome value $\mathrm{X}<-4.0$ and normalized chromosome value $\mathrm{Y}>4.0$, the sample was classified as male (XY).

6 . If condition 5 was met but normalized chromosome value $\mathrm{Y}$ was approximately two times greater than expected for the measured normalized chromosome value $\mathrm{X}$ value, the sample was classified as XYY. 
Table 3. Abnormal and Complex Karyotypes That Were Not Censored

\begin{tabular}{|c|c|c|}
\hline \multirow[b]{2}{*}{ Karyotype } & \multicolumn{2}{|c|}{$\begin{array}{c}\text { Massively Parallel } \\
\text { Sequencing Classification }\end{array}$} \\
\hline & Aneuploidy & Sex \\
\hline \multicolumn{3}{|l|}{ Monosomy X $(n=20)$} \\
\hline $45, X(n=15)$ & $\begin{array}{l}\text { Unaffected } \\
\qquad(21,18,13)\end{array}$ & Monosomy X \\
\hline $45, X(n=4)$ & $\begin{array}{l}\text { Unaffected } \\
\quad(21,18,13)\end{array}$ & Unclassified \\
\hline $45, X(n=1)$ & $\begin{array}{l}\text { Unaffected } \\
\qquad(21,18,13)\end{array}$ & Female \\
\hline \multicolumn{3}{|l|}{$\begin{array}{l}\text { Other autosomal trisomy or } \\
\text { partial trisomy }(n=5)\end{array}$} \\
\hline $47, \mathrm{XX},+16$ & $\begin{array}{c}\text { Chromosome } 16 \\
\text { aneuploidy }\end{array}$ & Unclassified \\
\hline $47, X X,+20$ & $\begin{array}{c}\text { Chromosome } 20 \\
\text { aneuploidy }\end{array}$ & Unclassified \\
\hline $\begin{array}{c}\text { Partial trisomy } 6 q 12 q 16.3 \\
\text { and } 6 q 16.3 \text {, no sex }\end{array}$ & $\begin{array}{l}\text { Unaffected } \\
\qquad(21,18,13)^{*}\end{array}$ & Female \\
\hline $47, X Y,+22$ & $\begin{array}{l}\text { Unaffected } \\
\quad(21,18,13)\end{array}$ & Male \\
\hline $47, X X,+22$ & $\begin{array}{l}\text { No fetal DNA } \\
\text { detected }\end{array}$ & \\
\hline \multicolumn{3}{|l|}{ Translocations $(n=7)$} \\
\hline Balanced $(n=6)$ & $\begin{array}{l}\text { Unaffected } \\
\qquad(21,18,13)\end{array}$ & $\begin{array}{c}\text { Correct class } \\
\text { (male or } \\
\text { female) }\end{array}$ \\
\hline Unbalanced $(\mathrm{n}=1)$ & $\begin{array}{l}\text { Unaffected } \\
\qquad(21,18,13)\end{array}$ & Female \\
\hline $\begin{array}{l}\text { Other complex mosaicism } \\
\qquad(\mathrm{n}=4)\end{array}$ & $\begin{array}{l}\text { Unaffected } \\
\qquad(21,18,13)\end{array}$ & $\begin{array}{c}\text { Correct class } \\
\text { (male or } \\
\text { female) }\end{array}$ \\
\hline $\begin{array}{l}\text { Other complex variants } \\
\qquad(\mathrm{n}=4)\end{array}$ & $\begin{array}{l}\text { Unaffected } \\
\qquad(21,18,13)\end{array}$ & $\begin{array}{c}\text { Correct class } \\
\text { (male or } \\
\text { female) }\end{array}$ \\
\hline
\end{tabular}

* An increased normalized chromosome value of 3.6 was noticed from sequencing tags in chromosome 6 after unblinding.

7. If the chromosome $\mathrm{X}$ and $\mathrm{Y}$ normalized chromosome values did not fit into any of the above criteria, the sample was classified as unclassified for sex.

Because the laboratory was blinded to the clinical information, the massively parallel sequencing results were not adjusted for any of the following demographic variables: maternal body mass index (calculated as weight $\left.(\mathrm{kg}) /[\text { height }(\mathrm{m})]^{2}\right)$, smoking status, presence of diabetes, types of conception (spontaneous or assisted), prior pregnancies, prior aneuploidy, or gestational age. Neither maternal nor paternal samples were used for classification, and the massively parallel sequencing classifications did not depend on the measurement of specific loci or alleles.

The sequencing results were returned to an independent contract biostatistician before unblinding and analysis. Personnel at the study sites, clinical research organizations (including the biostatistician generating random sampling lists), and the contract cytogeneticist were blinded to massively parallel sequencing results.

Statistical methods were documented in a detailed statistical analysis plan for the study. Point estimates for sensitivity and specificity along with exact 95\% CIs using the Clopper-Pearson method ${ }^{17}$ were computed for each of the six analytic categories. For all statistical estimation procedures performed, samples with no fetal DNA detected, censored for complex karyotype (per protocol-defined conventions), or unclassified by the sequencing test were removed.

\section{RESULTS}

Between June 2010 and August 2011, 2,882 pregnant women were enrolled in the study. The demographic characteristics of the eligible patients and the selected cohort are given in Table 1. Patients who enrolled and provided blood but were found during data monitoring to exceed inclusion criteria and have an actual gestational age at enrollment beyond $220 / 7$ weeks were allowed to remain in the study $(n=22)$. Three of these samples were in the selected set. Figure 2 shows the flow of samples between enrollment and analysis. There were 2,625 samples eligible for selection.

Per the random sampling plan, all eligible patients with an abnormal karyotype were selected for analysis (Fig. 2, Venn diagram) as well as a set of patients carrying euploid fetuses so that the total sequenced study population resulted in an approximately 4:1 ratio of unaffected to affected patients for trisomy 21. From this process, 534 patients were selected. Two samples subsequently were removed from analysis owing to sample-tracking issues in which a full chain of custody between sample tube and data acquisition did not pass quality audit (Fig. 2). This resulted in 532 patients for analysis contributed by 53 of the 60 study sites. The demographics of the selected cohort were similar to the overall cohort (Table 1).

Figure $3 \mathrm{~A}-\mathrm{C}$ shows the results for aneuploidy analysis of chromosomes 21, 18, and 13, and Figure $3 \mathrm{D}-\mathrm{F}$ shows the results for sex analysis. Table 4 shows the sensitivity, specificity, and CI for each of the six independent analyses, and Figures 4 and 5 show the graphical distribution of samples according to the normalized chromosome values after sequencing. In all six categories of analysis, 16 samples (3.0\%) were removed owing to no fetal DNA detected. After unblinding, there were no distinguishing clinical fea- 


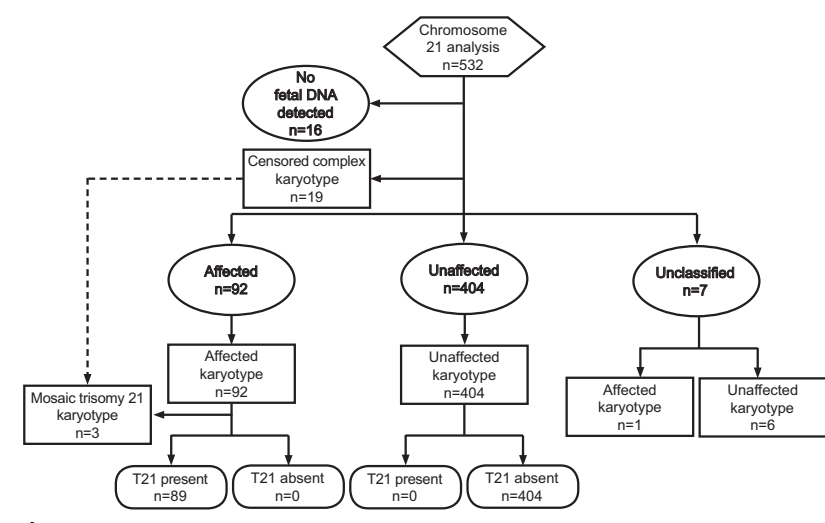

A

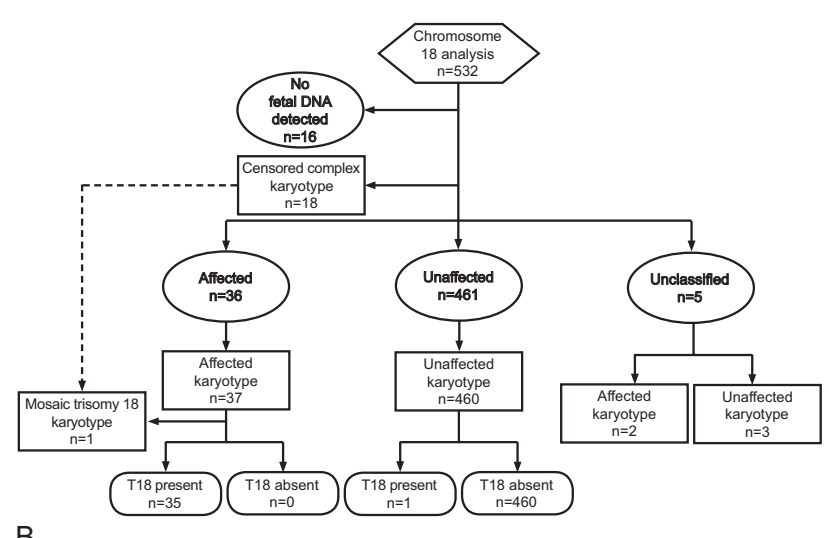

C
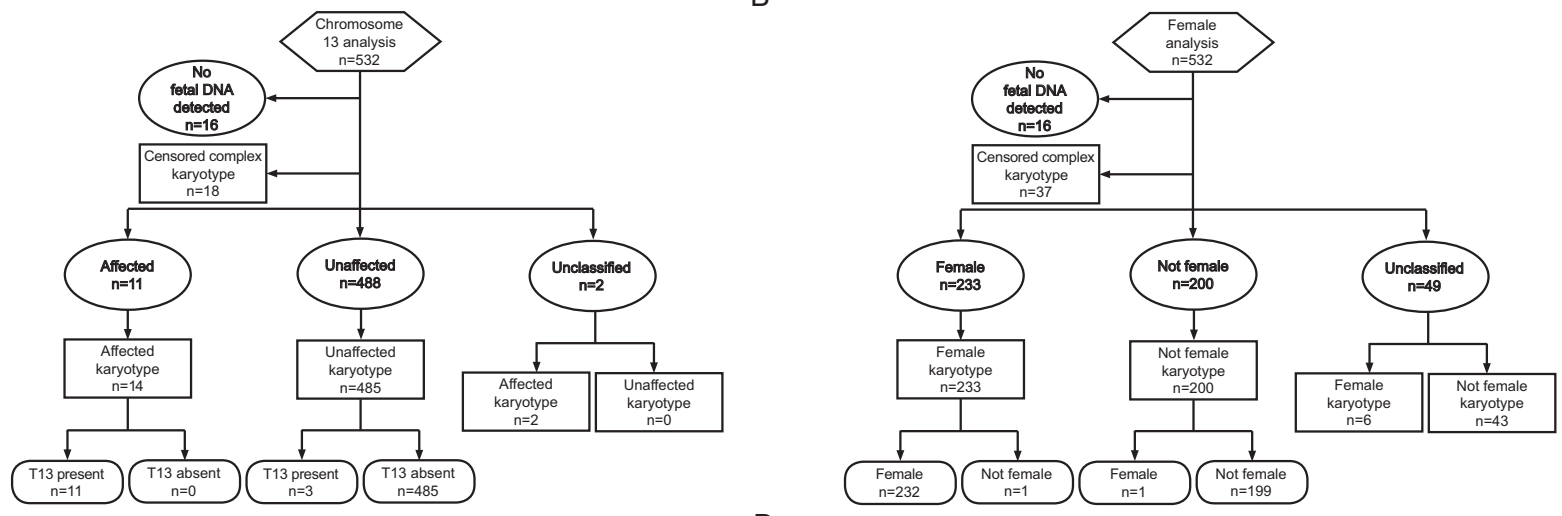

D

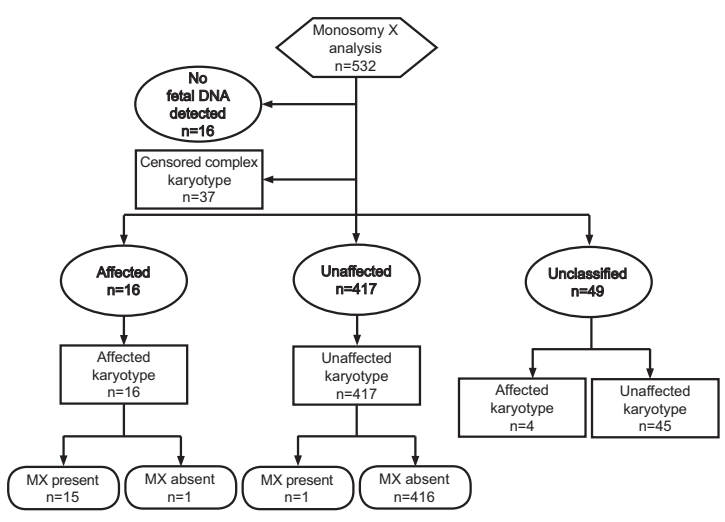

$\mathrm{F}$

Fig. 3. Analysis results. In all flow diagrams, ovals contain massively parallel sequencing results from the laboratory, rectangles contain karyotype results, and rectangles with rounded corners show comparative results used to determine test performance (sensitivity and specificity). The dashed lines in $\mathbf{A}$ and $\mathbf{B}$ denote the relationship between mosaic samples for T21 $(n=3)$ and T18 $(n=1)$ that were censored from the analysis of chromosome 21 and chromosome 18, respectively, but were correctly detected by massively parallel sequencing. A shows the results for chromosome 21, B shows the results for chromosome 18, C shows the results for chromosome 13, D shows the results for female classification, $\mathbf{E}$ shows the results for male classification, $\mathbf{F}$ shows the results for monosomy $\mathrm{X}$.

Bianchi. Genome-Wide Fetal Aneuploidy Detection. Obstet Gynecol 2012.

tures for these samples. The number of censored karyotypes for each category was dependent on the condition being analyzed (fully detailed in Fig. 3).

Sensitivity and specificity of the sequencing test to detect T2 1 in the analysis population $(\mathrm{n}=493)$ were $100 \%(95 \%$ CI 95.9-100.0) and 100\% (95\% CI
99.1-100.0), respectively (Table 4 and Fig. 3A). This included correct classification for one complex T21 karyotype, 47,XX, inv(7)(p22q32),+21, and two translocation T21 cases arising from Robertsonian translocations, one of which was also mosaic for monosomy X $(45, \mathrm{X},+21, \operatorname{der}(14 ; 21) \mathrm{q} 10 ; \mathrm{q} 10)[4] /$ 
Table 4. Massively Parallel Sequencing Performance

\begin{tabular}{lccrr}
\hline $\begin{array}{l}\text { Massively Parallel } \\
\text { Sequencing Performance }\end{array}$ & Sensitivity (\%) & 95\% Cl & Specificity (\%) & 95\% Cl \\
\hline Trisomy 21 $(\mathrm{n}=493)$ & $100.0(89 / 89)$ & $95.9-100.0$ & $100.0(404 / 404)$ & $99.1-100.0$ \\
Trisomy 18 $(\mathrm{n}=496)$ & $97.2(35 / 36)$ & $85.5-99.9$ & $100(460 / 460)$ & $99.2-100.0$ \\
Trisomy 13 $(\mathrm{n}=499)$ & $78.6(11 / 14)$ & $49.2-99.9$ & $100.0(485 / 485)$ & $99.2-100.0$ \\
Female $(\mathrm{n}=433)$ & $99.6(232 / 233)$ & 97.6 to more than 99.9 & $99.5(199 / 200)$ & 97.2 to more than 99.9 \\
Male $(\mathrm{n}=433)$ & $100.0(184 / 184)$ & $98.0-100.0$ & $100.0(249 / 249)$ & $98.5-100.0$ \\
Monosomy X $(\mathrm{n}=433)$ & $93.8(15 / 16)$ & $69.8-99.8$ & $99.8(416 / 417)$ & 98.7 to more than 99.9 \\
\hline
\end{tabular}

$\mathrm{Cl}$, confidence interval.

$46, X Y,+21, \operatorname{der}(14 ; 21) q 10 ; q 10)[17]$ and $46, X Y,+21$, $\operatorname{der}(21 ; 21) q 10 ; q 10)$. Sensitivity and specificity to detect $\mathrm{T} 18$ in the analysis population $(\mathrm{n}=496)$ were $97.2 \%$ (95\% CI 85.5-99.9) and 100\% (95\% CI 99.2-100.0) (Table 4 and Fig. 3B). Although censored (as per protocol) from the primary analysis, four samples with mosaic karyotypes for T21 and T18 were all correctly classified by massively parallel sequencing as "affected" for aneuploidy (Table 2). Because they were correctly detected they are indicated on the left side of Figure 3A and B. All remaining censored samples were correctly classified as unaffected for trisomies 21, 18, and 13 (Table 2). Sensitivity and specificity to detect T13 in

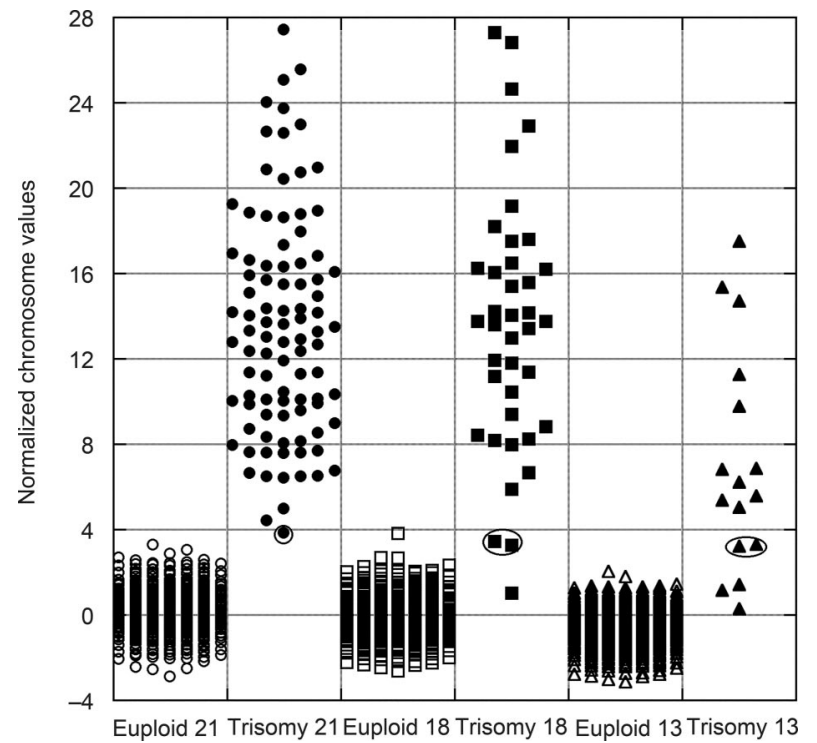

Fig. 4. Massively parallel sequencing normalized chromosome values compared with karyotype classifications for chromosomes 21, 18, and 13. Circles display classifications for chromosome 21, squares display classifications for chromosome 18, and triangles display classifications for chromosome 13. Unclassified samples with trisomy karyotypes have been circled.

Bianchi. Genome-Wide Fetal Aneuploidy Detection. Obstet Gynecol 2012. the analysis population were $78.6 \%(95 \%$ CI $49.2-$ 99.9) and $100 \%$ (95\% CI 99.2-100.0) (Fig. 3C). One T13 case detected arose from a Robertsonian translocation $(46, \mathrm{XY},+13, \operatorname{der}(13 ; 13) \mathrm{q} 10 ; \mathrm{q} 10)$.

There were seven unclassified samples in the chromosome 21 analysis (1.4\%), five in the chromosome 18 analysis $(1.0 \%)$, and two in the chromosome 13 analysis $(0.4 \%)$ (Fig. 3A-C). In all categories there was an overlap of three samples that had both a censored karyotype $(69, \mathrm{XXX})$ and no fetal DNA detected. One unclassified sample in the chromosome 21 analysis was correctly identified as T13 in the chromosome 13 analysis and one unclassified sample in the chromosome 18 analysis was correctly identified as T21 in the chromosome 21 analysis.

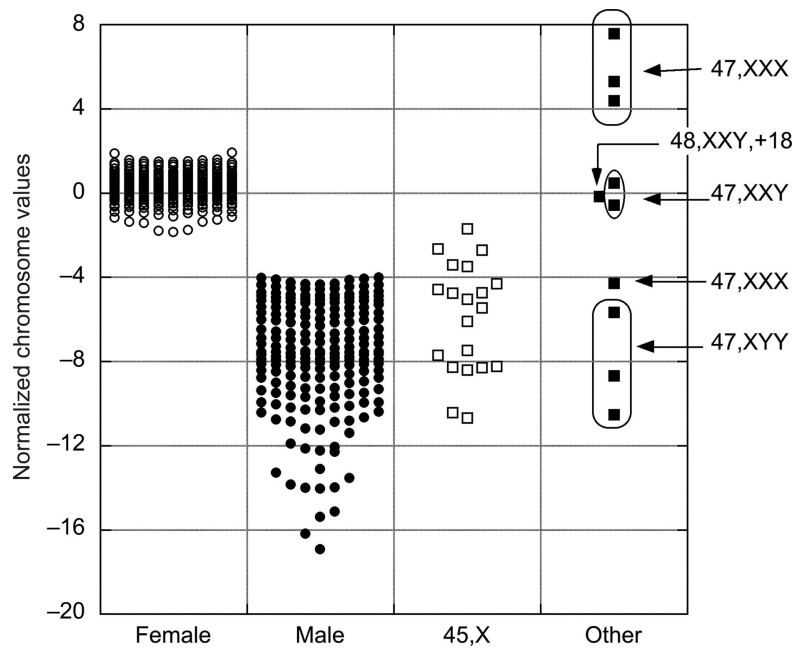

Fig. 5. Massively parallel sequencing normalized chromosome values (chromosome $X$ normalized chromosome value) compared with karyotype classifications for sex classifications. Empty circles are samples with female karyotypes, filled circles are samples with male karyotypes, and empty squares are samples with 45,X karyotypes. Other samples in the last column are labeled with the appropriate karyotype classification.

Bianchi. Genome-Wide Fetal Aneuploidy Detection. Obstet Gynecol 2012. 
The sex chromosome analysis population for determining performance of massively parallel sequencing (female, male, or monosomy X) was 433. Our refined algorithm for classifying the sex status, which allowed for accurate determination of sex chromosome aneuploidies, resulted in a higher number of massively parallel sequencing unclassified results. Sensitivity and specificity for detecting diploid female state $(\mathrm{XX})$ were $99.6 \%$ (95\% CI 97.6 to more than 99.9$)$ and $99.5 \%$ (95\% CI 97.2 to more than 99.9), respectively; sensitivity and specificity to detect male (XY) were both 100\% (95\% CI 98.0-100.0); and sensitivity and specificity for detecting monosomy $\mathrm{X}$ $(45, \mathrm{X})$ were $93.8 \%(95 \%$ CI $69.8-99.8)$ and $99.8 \%$ (95\% CI 98.7 to more than 99.9) (Fig. 3D-F). Although censored from the analysis (as per protocol), the massively parallel sequencing classifications of mosaic monosomy $\mathrm{X}$ karyotypes were as follows (Table 2): two of seven classified as monosomy X, three of seven with a $\mathrm{Y}$ chromosome component classified as $\mathrm{XY}$, and two of seven with an $\mathrm{XX}$ chromosome component classified as female. Two samples that were classified by massively parallel sequencing as monosomy $\mathrm{X}$ had karyotypes of 47,XXX and 46,XX. Eight of ten sex chromosome aneuploidies for karyotypes 47,XXX, 47,XXY and $47, \mathrm{XYY}$ were correctly classified (Table 2). If we had limited the sex chromosome classifications to monosomy $\mathrm{X}, \mathrm{XY}$ and $\mathrm{XX}$, most of the unclassified samples would have been correctly classified as male, but we would not have identified the XXY and XYY sex aneuploidies.

In addition to accurately classifying trisomies 21 , 18,13 and sex, the sequencing results also prospectively correctly classified aneuploidy for chromosomes 16 and 20 in two samples $(47, \mathrm{XX},+16$ and $47, \mathrm{XX},+20)$ (Table 3). Chromosome 22 aneuploidy was not detected by massively parallel sequencing in two other patients with trisomy 22 (one of these had no fetal DNA detected). Interestingly, one sample with a complex karyotype involving the long arm of chromosome 6 (6q) and two duplications, one of which was $37.5 \mathrm{Mb}$ in size, showed an increased normalized chromosome value from sequencing tags in chromosome 6 (normalized chromosome value $=3.6$ ). Two prior articles have shown that detection of partial chromosome deletion is feasible. ${ }^{11,18} \mathrm{In}$ another sample, aneuploidy of chromosome 2 was detected by massively parallel sequencing but not observed in the fetal karyotype at amniocentesis $(46, \mathrm{XY})$. Other complex karyotype variants shown in Tables 2 and 3 include samples from fetuses with chromosome inversions, deletions, translocations, triploidy, and other abnormalities that were not detected here but potentially could be classified by massively parallel sequencing at higher sequencing density or with further algorithm optimization or both. In these cases, massively parallel sequencing correctly classified the samples as unaffected for trisomy 21,18 , or 13 and as male or female.

We also examined specific clinical variables that might affect massively parallel sequencing performance. In this study, 38 of 532 analyzed samples were from women who underwent assisted reproduction. Of these 38, 17 had chromosomal abnormalities; no false-positive or false-negative results were detected in this subpopulation.

\section{DISCUSSION}

This prospective study to determine the capability of massively parallel sequencing to detect whole chromosome fetal aneuploidy from maternal plasma was designed to emulate the real world scenario of sample collection, processing and analysis. Whole blood samples were obtained at the enrollment sites, did not require immediate processing, and were shipped overnight to the sequencing laboratory. In contrast to a prior prospective study that only involved chromosome $21,{ }^{15}$ in this study, all eligible samples with any abnormal karyotype were sequenced. The sequencing laboratory did not have prior knowledge of which fetal chromosomes might be affected nor the ratio of aneuploid to euploid samples. The study design recruited a high-risk study population of pregnant women to assure a statistically significant prevalence of aneuploidy, and Tables 2 and 3 indicate the complexity of the karyotypes that were analyzed. The results demonstrate that: 1) fetal aneuploidies (including those resulting from translocation trisomy, mosaicism, and complex variations) can be detected with high sensitivity and specificity and 2) aneuploidy in one chromosome does not affect the ability of the massively parallel sequencing method to correctly identify the euploid status of other chromosomes. The algorithms used in the previous studies appear to be unable to effectively determine other aneuploidies that inevitably would be present in a general clinical population. ${ }^{10,14}$

With regard to mosaicism, the massively parallel sequencing analysis in this study was able to classify samples as aneuploidy that had mosaic karyotypes for chromosomes 21 and 18 in four of four affected samples. These results demonstrate the sensitivity of the massively parallel sequencing analysis for detecting specific characteristics of cell free DNA in a complex mixture. In one case, the massively parallel 
sequencing data for chromosome 2 indicated a whole or partial chromosome aneuploidy whereas the amniocentesis karyotype result for chromosome 2 was diploid. In two other examples, one sample with 47 , XXX karyotype and another with a 46, XX karyotype, massively parallel sequencing classified these samples as monosomy $\mathrm{X}$. It is possible these are mosaic cases, or that the pregnant woman herself is mosaic. (It is important to remember that the sequencing is performed on total DNA, which is a combination of maternal and fetal DNA.) Although cytogenetic analysis of amniocytes or villi from invasive procedures is currently the reference standard for aneuploidy classification, a karyotype performed on a limited number of cells cannot rule out low-level mosaicism. The current clinical study design did not include long-term infant follow-up or access to placental tissue at delivery, so we are unable to determine whether these were true-positive or false-positive results. We speculate that the specificity of the sequencing process, coupled with optimized algorithms to detect genome wide variation, may ultimately provide more sensitive identification of fetal DNA abnormalities, particularly in cases of mosaicism, than standard karyotyping.

The International Society for Prenatal Diagnosis has issued a Rapid Response Statement commenting on the commercial availability of massively parallel sequencing for prenatal detection of Down syndrome. ${ }^{19}$ They state that before routine massively parallel sequencing-based population screening for fetal Down syndrome is introduced, evidence is needed that the test performs in some subpopulations, such as in women who conceive by in vitro fertilization. The results reported here suggest that massively parallel sequencing is accurate in this group of pregnant women, many of whom are at high risk for aneuploidy.

Although these results demonstrate the excellent performance of massively parallel sequencing with optimized algorithms for aneuploidy detection across the genome in singleton pregnancies from women at increased risk for aneuploidy, more experience, particularly in low-risk populations, is needed to build confidence in the diagnostic performance of the method when the prevalence is low and in multiple gestation. In the early stages of clinical implementation, massively parallel sequencing for chromosomes 21,18 , and 13 should be used after a positive firsttrimester or second-trimester screening result. This will reduce unnecessary invasive procedures caused by the false-positive screening results, with a concomitant reduction in procedure related adverse events.
Invasive procedures could be limited to confirmation of a positive result from sequencing. We acknowledge, however, that there are certain clinical scenarios (eg, advanced maternal age and infertility) in which pregnant women will want to avoid an invasive procedure; they may request this test as an alternative to the primary screen or invasive procedure or both. All patients should receive thorough pretest counseling to ensure that they understand the limitations of the test and the implications of the results. As experience accumulates with more samples, it is possible that this test will replace current screening protocols and become a primary screening and ultimately a noninvasive diagnostic test for fetal aneuploidy.

\section{REFERENCES}

1. Milunsky A, Milunsky JM. Genetic disorders and the fetus: diagnosis, prevention, and treatment. 6th ed. Hoboken (NJ): Wiley-Blackwell; 2010.

2. Malone FD, Canick JA, Ball RH, Nyberg DA, Comstock CH, Bukowski R, et al. First-trimester or second-trimester screening, or both, for Down's syndrome. N Engl J Med 2005;353: 2001-11.

3. Fan HC, Blumenfeld YJ, Chitkara U, Hudgins L, Quake SR. Noninvasive diagnosis of fetal aneuploidy by shotgun sequencing DNA from maternal blood. Proc Natl Acad Sci U S A 2008; 105:16266-71.

4. Fan HC, Quake SR. Sensitivity of noninvasive prenatal detection of fetal aneuploidy from maternal plasma using shotgun sequencing is limited only by counting statistics. PLoS One 2010;5:e10439.

5. Fan HC, Blumenfeld YJ, Chitkara U, Hudgins L, Quake SR. Analysis of the size distributions of fetal and maternal cell-free DNA by paired-end sequencing. Clin Chem 2010;56: 1279-86.

6. Chiu RW, Chan KC, Gao Y, Lau VY, Zheng W, Leung TY, et al. Noninvasive prenatal diagnosis of fetal chromosomal aneuploidy by massively parallel genomic sequencing of DNA in maternal plasma. Proc Natl Acad Sci U S A 2008;105: $20458-63$.

7. Chiu RW, Cantor CR, Lo YM. Non-invasive prenatal diagnosis by single molecule counting technologies. Trends Genet 2009;25:324-31.

8. Lo YM, Chan KC, Sun H, Chen EZ, Jiang P, Lun FM, et al Maternal plasma DNA sequencing reveals the genome-wide genetic and mutational profile of the fetus. Sci Transl Med 2010;2:61ra91.

9. Chiu RW, Lo YM. Non-invasive prenatal diagnosis by fetal nucleic acid analysis in maternal plasma: the coming of age. Semin Fetal Neonatal Med 2011;16:88-93.

10. Ehrich M, Deciu C, Zwiefelhofer T, Tynan JA, Cagasan L, Tim $\mathrm{R}$, et al. Noninvasive detection of fetal trisomy 21 by sequencing of DNA in maternal blood: a study in a clinical setting. Am J Obstet Gynecol 2011;204:205.e1-11.

11. Sehnert AJ, Rhees B, Comstock D, de Feo E, Heilek G, Burke $\mathrm{J}$, et al. Optimal detection of fetal chromosomal abnormalities by massively parallel DNA sequencing of cell-free fetal DNA from maternal blood. Clin Chem 2011;57:1042-9. 
12. Chen EZ, Chiu RW, Sun H, Akolekar R, Chan KC, Leung TY, et al. Noninvasive prenatal diagnosis of fetal trisomy 18 and trisomy 13 by maternal plasma DNA sequencing. PLoS One 2011;6:e21791.

13. Lo D, Chiu RW. Plasma nucleic acid analysis by massively parallel sequencing: pathological insights and diagnostic implications. J Pathol 2011;225:318-23.

14. Chiu RW, Akolekar R, Zheng YW, Leung TY, Sun H, Chan $\mathrm{KC}$, et al. Non-invasive prenatal assessment of trisomy 21 by multiplexed maternal plasma DNA sequencing: large scale validity study. BMJ 2011;342:c7401.

15. Palomaki GE, Kloza EM, Lambert-Messerlian GM, Haddow JE, Neveux LM, Ehrich M, et al. DNA sequencing of maternal plasma to detect Down syndrome: An international clinical validation study. Genet Med 2011:913-20.
16. Kidd KK, Pakstis AJ, Speed WC, Grigorenko EL, Kajuna SL, Karoma NJ, et al. Developing a SNP panel for forensic identification of individuals. Forensic Sci Int 2006;164: $20-32$.

17. Clopper C, Pearson ES. The use of confidence or fiducial limits illustrated in the case of the binomial. Biometrika 1934;26:404-13.

18. Peters D, Chu T, Yatsenko SA, Hendrix N, Hogge WA, Surti $\mathrm{U}$, et al. Noninvasive prenatal diagnosis of a fetal microdeletion syndrome. N Engl J Med 2011;365:1847-8.

19. Benn P, Borrell A, Cuckle H, Dugoff L, Gross S, Johnson JA, et al. Prenatal detection of Down syndrome using Massively Parallel Sequencing (MPS): a rapid response statement from a committee on behalf of the Board of the International Society for Prenatal Diagnosis, 24 October 2011. Prenat Diagn 2012 Jan 24 [epub ahead of print]. 
000 Genome-Wide Fetal Aneuploidy Detection by Maternal Plasma DNA Sequencing

This prospective study demonstrates that massively parallel sequencing of maternal plasma DNA is effective for detecting fetal aneuploidy for multiple chromosomes across the genome.

Diana W. Bianchi, Lawrence D. Platt, James D. Goldberg, Alfred Z. Abuhamad, Amy J. Sehnert, Richard P. Rava, on behalf of the MatErnal BLood IS Source to Accurately diagnose fetal aneuploidy (MELISSA) Study Group 